\title{
LINE WIDTH IN MAGNETIC RESONANCE DUE TO EMISSION OF RADIATION
}

\author{
K.N. SHRIVASTAVA \\ Department of Physics, University of California, Santa Barbara, California 93106, USA \\ and Fysische Laboratorium*, Rijksuniversiteit, Utrecht, The Netherlands
}

Received 15 October 1975

\begin{abstract}
Line width in ferromagnetic resonance is found to consist of the magnon scattering, the superradiance, and a new effect due to the simultaneous presence of magnon scattering and the superradiance.
\end{abstract}

We wish to present the line width of a uniform magnon in ferromagnetic resonance arising from the superradiance and a new process arising from the noncommutation between the superradiance and the magnon-magnon surface scattering.

Consider a one-magnon-one-photon interaction [1] arising from the $x$-component of the magnetic vector of the electromagnetic field and the magnetization, $g \beta h_{x} \cdot S_{x}$, and a magnon-magnon surface pit scattering [2], so that the Hamiltonian of the system assumes the form

$$
\begin{aligned}
& \mathscr{X}=\mathscr{X}_{0}+\mathscr{X}_{1}+\mathscr{X}_{2} \\
& \mathscr{X}_{0}=\hbar \omega_{0} b_{0}^{\dagger} b_{0}+\hbar \Omega_{0} a^{\dagger} a_{0}+\sum_{k}^{\prime} \hbar \Omega_{k} a_{k}^{\dagger} a_{k} \\
& \mathscr{X}_{1}=i \alpha\left(b_{0}^{\dagger}-b_{0}\right)\left(a_{0}+a_{0}^{\dagger}\right) \\
& \mathscr{X}_{2}=\sum_{k}^{\prime} F_{k}\left(a_{0}^{\dagger} a_{k}+a_{0} a_{k}^{\dagger}\right)
\end{aligned}
$$

where the coupling constant $\alpha$ in the free space is $\left(\pi \hbar M_{0} V \hbar \omega_{0}\right)^{1 / 2}$ whereas, in the wave guide, $\alpha=\left[3 \hbar M_{0} V \hbar \omega_{0} \lambda^{3} /\left(2 a b \lambda_{\mathrm{g}}\right)\right]^{1 / 2} \cos k_{\mathrm{g}} l$

where $M_{0}$ is the magnetization, $V$ is the volume of the sample, $\omega_{0}$ the unperturbed photon frequency, $\lambda$ the wave length in the free space and $\lambda_{g}$ in the guide and $l$ are the distance of the sample from the terminating end of the guide of area of cross section $a b$; $F_{k}$ is the structure factor in the coupling between the uniform magnon and finite wave vector magnon at the surface of the sample inside a dipotar spread band. $a_{0, k}^{\dagger}$ and $a_{0, k}$ are the magnon creation and destruc-

\footnotetext{
* Present address.
}

tion operators while $b_{0}^{\dagger}$ and $b_{0}$ are the photon creation and annihilation operators.

We calculate the equation of motion of the Green function of the uniform magnon and the related oneparticle Green functions which after taking the Fourier transform are found to be

$$
\begin{aligned}
& \left.\left(E-\hbar \Omega_{0}\right)\left\langle a_{0} \mid a_{0}^{\dagger}\right\rangle\right\rangle=\frac{\hbar}{2 \pi}-\mathrm{i} \alpha\left\langle\left\langle b_{0} \mid a_{0}^{\dagger}\right\rangle\right\rangle \\
& +\mathrm{i} \alpha\left\langle\left\langle b_{0}^{\dagger} \mid a_{0}^{\dagger}\right\rangle\right\rangle+\sum_{k}^{\prime} F_{k}\left\langle\left\langle a_{k} \mid a_{0}^{\dagger}\right\rangle\right\rangle \\
& \left(E-\hbar \omega_{0}\right)\left\langle\left\langle b_{0} \mid a_{0}^{\dagger}\right\rangle\right\rangle=\mathrm{i} \alpha\left\langle\left\langle a_{0} \mid a_{0}^{\dagger}\right\rangle+\mathrm{i} \alpha\left\langle\left\langle a_{0}^{\dagger} \mid a_{0}^{\dagger}\right\rangle\right\rangle\right. \\
& \left(E+\hbar \omega_{0}\right)\left\langle\left\langle b_{0}^{\dagger} \mid a_{0}^{\dagger}\right\rangle\right\rangle=\mathrm{i} \alpha\left\langle\left\langle a_{0}^{\dagger} \mid a_{0}^{\dagger}\right\rangle+\mathrm{i} \alpha\left\langle\left\langle a_{0} \mid a_{0}^{\dagger}\right\rangle\right\rangle\right. \\
& \left(E-\hbar \Omega_{k}\right)\left\langle\left\langle a_{k} \mid a_{0}^{\dagger}\right\rangle\right\rangle=\sum_{k}^{\prime} F_{k}\left\langle\left\langle a_{0} \mid a_{0}^{\dagger}\right\rangle\right\rangle \\
& \left(E+\hbar \Omega_{k}\right)\left\langle\left\langle a_{k}^{\dagger} \mid a_{0}^{\dagger}\right\rangle\right\rangle=-\sum_{k}^{\prime} F_{k}\left\langle\left\langle a_{0}^{\dagger} \mid a_{0}^{\dagger}\right\rangle\right. \\
& \left(E-\hbar \Omega_{0}\right)\left\langle\left\langle a_{0}^{\dagger} \mid a_{0}^{\dagger}\right\rangle\right\rangle=-\mathrm{i} \alpha\left\langle\left\langle b_{0}^{\dagger} \mid a_{0}^{\dagger}\right\rangle\right\rangle \\
& +\mathrm{i} \alpha\left\langle\left\langle b_{0} \mid a_{0}^{\dagger}\right\rangle\right\rangle-\sum_{k} F_{k}\left\langle\left\langle a_{k}^{\dagger} \mid a_{0}^{\dagger}\right\rangle\right\rangle
\end{aligned}
$$

Simultaneous solution of above gives the one-uniform magnon Green function as

$\left(E-\hbar \Omega_{0}-\Sigma\right)\left\langle\left\langle a_{0} \mid a_{0}^{\dagger}\right\rangle\right\rangle=\hbar / 2 \pi$

with the self-energy of the uniform magnon given by 


$$
\begin{aligned}
\Sigma & =\sum_{k}^{\prime} \frac{F_{k}^{2}}{E-\hbar \Omega_{k}}-\alpha^{2}\left(\frac{1}{\frac{E+\hbar \omega_{0}}{E-\hbar \omega_{0}}}-\frac{1}{E}\right)^{2} \\
& -\alpha^{4}\left(\frac{1}{E+\hbar \omega_{0}}-\frac{1}{E-\hbar \omega_{0}}\right) \\
& \times\left[E+\hbar \Omega_{0}-\sum_{k}^{\prime} \frac{F_{k}^{2}}{E+\hbar \Omega_{k}}\right. \\
& \left.-\alpha^{2}\left(\frac{1}{E+\hbar \omega_{0}}-\frac{1}{E-\hbar \omega_{0}}\right)\right]^{-1} .
\end{aligned}
$$

The life time of the uniform magnon is determined by $1 / \tau=-(2 / \hbar) \operatorname{Im} \Sigma$, where the imaginary part of the self energy is defined by Dirac's identity, $\lim _{\epsilon \rightarrow 0} 1 /(x \pm \mathrm{i} \epsilon)=(P / x) \mp \mathrm{i} \pi \delta(x)$. The dominant contribution to the line width is then found to be $1 / \tau=$ $\Delta H=\Delta H_{1}+\Delta H_{2}+\Delta H_{3}$

where

$$
\begin{aligned}
& \Delta H_{1}=\frac{2 \pi}{\hbar} \sum_{k}^{\prime} F_{k}^{2} \delta\left(E-\hbar \Omega_{k}\right) \approx \frac{2 \pi}{\hbar} \int_{0}^{k_{\max }}\left|F_{k}\right|^{2} \rho_{k} \mathrm{~d} k \\
& \Delta H_{2}=\frac{2 \pi}{\hbar} \frac{\alpha^{2} \omega^{2}}{2 \pi^{2} \hbar c^{3}} \\
& \Delta H_{3}=\frac{2 \pi}{\hbar} \sum_{k}^{\prime} \alpha^{2} F_{k}^{2}\left[\frac{\delta\left(E-h \Omega_{k}\right)}{\left(\hbar \omega_{0}+\hbar \Omega_{k}\right)\left(\hbar \Omega_{k}+\hbar \Omega_{0}\right)}\right.
\end{aligned}
$$

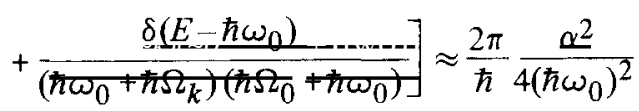

$$
\begin{aligned}
& \times \int_{0}^{k_{\max }}\left|F_{k}\right|^{2} \rho_{k} \mathrm{~d} k+\frac{2 \pi \alpha^{2}}{\hbar} \frac{\omega^{2}}{2 \pi^{2} \hbar c^{3}} \frac{\left|F_{k}\right|^{2}}{4\left(\hbar \omega_{0}\right)^{2}} \text {. }
\end{aligned}
$$

Here (10) is the result of Sparks et al. [2] for the twomagnon surface pit scattering. The next term (11) has been calculated by introducing a microwave slit width giving rise to a density of states for the photon of $\omega^{2} / 2 \hbar \pi^{2} c^{3}$. This term can be interpreted to arise from Dicke's superradiance. The next term (12) arises because of the noncommutation of superradiance and the magnon-magnon surface scattering. For $\mathrm{MnF}_{2}$ under normal circumstances [3], the (10) can vary from about 2 Gauss for a polished crystal to about 50 Gauss for a rough sample, (11) is about 15 Gauss and $(12)$ is about $\lesssim 4$ Gauss.

Thus, we find that interesting new effects occur owing to the simultaneous presence of superradiance and the magnon scattering.

I am grateful to Professors V. Jaccarino, Daniel Hone, Douglas J. Scalapino and Robert M. White for discussion. Thanks are due to Professor C. Kittel and Dr. Ernst Schloemann for comments.

\section{References}

[1] H. LeGall, B. Desormiere and E. Milot, J. Phys. Chem.' Solids 30 (1969) 979, 1135.

[2] M. Sparks, R. Loudon and C. Kittel, Phys. Rev. 122 (1961) 791 ;

P. Pincus, M. Sparks and R.C. LeCraw 124 (1961) 1015.

[3] R.W. Sanders, D. Paquette, V. Jaccarino and S. Rezende, Phys. Rev. B10 (1974) 132. 